\title{
Three-Level Optical Stark Effect of Excitons in GaAs Cylindrical Quantum Wires
}

\author{
Dinh Nhu Thao $\mathbb{D}^{1},{ }^{1}$ Duong Dinh Phuoc $\mathbb{D},{ }^{1}$ Le Thi Ngoc Bao $\mathbb{D},{ }^{2}$ Le Thi Dieu Hien $\mathbb{D},{ }^{1,2}$ \\ Tran Phan Thuy Linh $\mathbb{D}^{3},{ }^{3}$ and Nguyen Thi Le Thuy ${ }^{4}$ \\ ${ }^{1}$ Hue University of Education, Hue University, 34 Le Loi Street, Hue City, Vietnam \\ ${ }^{2}$ Hue University of Sciences, Hue University, 77 Nguyen Hue Street, Hue City, Vietnam \\ ${ }^{3}$ Faculty of Physics, Hanoi National University of Education, 136 Xuanthuy, Caugiay, Hanoi 100000, Vietnam \\ ${ }^{4}$ Research Laboratories of Saigon Hi-Tech Park, Lot I3, Road N2, Saigon Hi-Tech Park, District 9, Ho Chi Minh City, Vietnam
}

Correspondence should be addressed to Dinh Nhu Thao; dnthao@hueuni.edu.vn

and Duong Dinh Phuoc; dinhphuoc2808@gmail.com

Received 8 January 2021; Revised 25 February 2021; Accepted 3 March 2021; Published 17 March 2021

Academic Editor: Mai Duy Hien

Copyright ( 2021 Dinh Nhu Thao et al. This is an open access article distributed under the Creative Commons Attribution License, which permits unrestricted use, distribution, and reproduction in any medium, provided the original work is properly cited.

\begin{abstract}
This study looks at the three-level optical Stark effect of excitons in GaAs cylindrical quantum wires, utilizing the renormalized wave function theory. By applying the three-level model consisting of the first two electron levels connected via a powerful pump laser and the first hole level, we observe the appearance of the excitonic optical Stark effect through the appearance of two separated peaks in the exciton absorption spectra. In addition, the strong impact of the pump laser detuning and the wire radius on the optical Stark effect are also put under thorough examination. Finally, a brief guidance for experimental verification is also suggested.
\end{abstract}

\section{Introduction}

Low-dimensional semiconductor structures, which are the core of many semiconductor devices, have been studied for new applied fields thanks to the inventions of advanced technologies [1-12]. These devices play important roles in the fields of medicine, military, telecommunication, and many others [13-17]. The semiconductor quantum wires, one of the lowdimensional structures that possess many special properties, have drawn the attention of scientists [18-22]. These structures could be applied to create high-speed lasers [23, 24], quantum electron waveguides [25], resonant tunneling field-effect transistors [26, 27], or telecommunication networks [28]. Recently, studies of movement and interaction among electrons in the quantum wires have shown the quantum effects that can lead to various applications in the new quantum technology [29]. Nonetheless, many new physical properties of these structures, which are expected to have more breakthrough applications in the future, have been little known to researchers.

In low-dimensional semiconductor systems, scientists have observed the excitonic optical Stark effect when irradiat- ing a strong pump laser pulse in $[30,31]$. This effect resulted from the interaction between exciton states, displayed through splitting and shifting the absorption spectrum of the exciton [32-35]. Based on these properties, the researchers proposed a number of prospective applications of the excitonic optical Stark effect, such as making optical switching devices $[33,34]$, optical modulators [35], mesoporous hybrid multifunctional system [36], or optically controlled field-effect transistors [37]. So far, the excitonic optical Stark effect has been primarily studied on quantum wells and quantum dots [30,31]. The existence of this effect in quantum wire structures is still under investigation.

The excitonic optical Stark effect is divided into two types. The first type is the consequence of a coupling of the exciton ground state and an exciton-excited state, e.g., the first exciton state under the effect of a strong pump laser beam called a two-level Stark effect [38]. The second type is due to a coupling of two exciton-excited states under the effect of a pump laser beam of lower intensity, known as a three-level Stark effect $[38,39]$. The latter has more applicability because it is more likely to occur. The excitonic optical 
Stark effect can be experimentally studied by the pump-probe technique $[40,41]$. For theoretical research, methods to be deployed can be the renormalized wave function theory [42], the density matrix approach $[43,44]$, the finite difference method [45], the photoemission theory [46], or the nonequilibrium many body perturbation theory [47]. In our viewpoint, the renormalized wave function formulation has many advantages such as it only renormalizes the wave function of exciton, or it is easy to confirm the existence of the effect as well as to reveal its physical properties.

In this study, we investigate the three-level excitonic optical Stark effect in GaAs cylindrical quantum wires utilizing the renormalized wave function formulation. We have also successfully applied that formulation to study the excitonic quantum beats [48], a phenomenon that occurs as the consequence of the three-level excitonic optical Stark effect in the same structures of quantum wires. Therefore, both the recent report and Ref. [48] share the same starting point. Hence, we can expect to successfully study the three-level excitonic optical Stark effect in those structures. This paper is organized into three main sections, with the present section justifying the rationale and introducing the stated problem. Section 2 provides the formulation and basic equations. Section 3 presents and discusses the findings of the study, based on which, conclusions are arrived at.

\section{Formulation}

2.1. Wave Functions and Quantized Levels of Electron and Hole in a Cylindrical Quantum Wire. Let consider a circular cylindrical quantum wire in the cylindrical coordinates $(r, \varphi, z)$. Suppose that the quantum wire is of the radius $R$ and stays along the $O z$ axis, and the particles are confined in the plane $O x y$ by an infinite cylindrical symmetric potential,

$$
U(x, y)=U(r)= \begin{cases}0, & r \leq R, \\ \infty, & r>R,\end{cases}
$$

where $r$ is the length from the wire axis to the considered position. The envelope wave functions describing the states of the electron and hole in the confined plane can be written as $[48,49]$

$$
\Psi_{m n}^{e, h}(\vec{r})=\frac{1}{\sqrt{\pi R^{2}}} \frac{J_{m}\left(\chi_{m n}(r / R)\right)}{J_{m+1}\left(\chi_{m n}\right)} e^{i m \varphi},
$$

where $\vec{r}=(r, \varphi)$ is the position vector of the particle, $J_{m}(r)$ are the Bessel functions of the first kind of order $m(m=0,1,2, \cdots)$, and $\chi_{m n}$ are its $n$th zero points $(n=1,2, \cdots)$. The full wave functions of the electron and hole are presented as

$$
\Lambda_{m n}^{e, h}(\vec{r})=u_{c, v}(\vec{r}) \Psi_{m n}^{e, h}(\vec{r})
$$

where $u_{c, v}(\vec{r})$ is the periodic Bloch function in the first Brillouin zone and $c$ and $v$ signify the conduction and valence band, respectively.

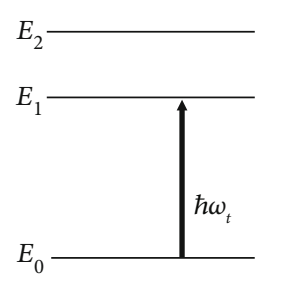

(a)

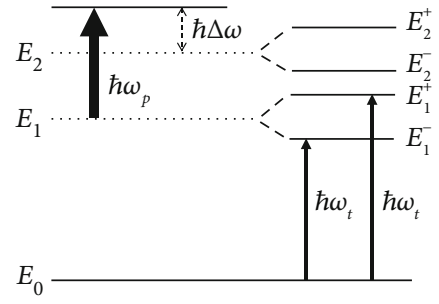

(b)
FIgURe 1: The three-level diagram of energy: (a) Under no pump laser, the system contains one hole level $\left(E_{0}\right)$ and two electron levels $\left(E_{1}\right.$ and $\left.E_{2}\right)$. The probe laser of photon energy $\hbar \omega_{t}$ detects the interband transition between the $E_{0}$ and $E_{1}$ levels (thin arrow). (b) Under the pump laser of photon energy $\hbar \omega_{p}$ (thick arrow), level $E_{1}$ is separated into levels $E_{1}^{-}$and $E_{1}^{+}$; level $E_{2}$ is separated into levels $E_{2}^{-}$and $E_{2}^{+}$. The probe laser of photon energy $\hbar \omega_{t}$ detects two interband transitions from $E_{0}$ level to $E_{1}^{-}$level and from $E_{0}$ level to $E_{1}^{+}$level (thin arrows).

The quantized levels of the electron and hole are determined as

$$
\left\{\begin{array}{l}
\varepsilon_{m n}^{e}=E_{g}+\frac{\hbar^{2} \chi_{m n}^{2}}{2 m_{e} R^{2}}, \\
\varepsilon_{m n}^{h}=\frac{\hbar^{2} \chi_{m n}^{2}}{2 m_{h} R^{2}}
\end{array}\right.
$$

where $m_{e}$ and $m_{h}$ are the effective masses of the electron and hole, respectively, and $E_{g}$ is the energy gap of the wire material. Here, we set the top of the valence band as the origin for calculating the energy of the particles.

In this paper, we use a three-level energy diagram to study the excitonic optical Stark effect in the quantum wire. Therefore, we investigate a three-level model in which $E_{0}$ is the first hole level, while $E_{1}$ and $E_{2}$ are the first two electron ones (as shown in Figure 1(a)).

The energies of the particles are determined as follows [48]:

$$
\left\{\begin{array}{l}
E_{0} \equiv \varepsilon_{01}^{h}, \\
E_{1} \equiv \varepsilon_{01}^{e}, \\
E_{2} \equiv \varepsilon_{11}^{e},
\end{array}\right.
$$

with the compatible time-dependent wave functions

$$
\left\{\begin{array}{l}
\Pi_{0}(\vec{r}, t)=\Pi_{0}(\vec{r}) e^{-(i / \hbar) E_{0} t}, \\
\Pi_{1}(\vec{r}, t)=\Pi_{1}(\vec{r}) e^{-(i / \hbar) E_{1} t}, \\
\Pi_{2}(\vec{r}, t)=\Pi_{2}(\vec{r}) e^{-(i / \hbar) E_{2} t},
\end{array}\right.
$$


in which $\Pi_{j}(\vec{r})(j=0,1,2)$ are the stationary wave functions of the particles in the quantum wire and are defined by [48]

$$
\left\{\begin{array}{l}
\Pi_{0}(\vec{r}) \equiv \Lambda_{01}^{h}(\vec{r}), \\
\Pi_{1}(\vec{r}) \equiv \Lambda_{01}^{e}(\vec{r}), \\
\Pi_{2}(\vec{r}) \equiv \Lambda_{11}^{e}(\vec{r}) .
\end{array}\right.
$$

2.2. Electronic Wave Function and Energy Levels in the Presence of Pump Laser. To seek the evidence of the existence of the excitonic optical Stark effect in the quantum wire and study its properties, we irradiate concurrently two lasers on the system. A strong pump laser, which resonates with the energy separation between two electron levels, is utilized to stimulate the intraband transition of the electron (as indicated by the thick arrow in Figure 1(b)). Besides, a weak probe laser is utilized to identify the interband transitions among the hole level and the electron levels (as indicated by the thin arrow in Figure 1(b)). The form of the laser waves is chosen as follows:

$$
\vec{E}(t)=\vec{n} A_{x} e^{-i \omega_{x} t},
$$

where $\vec{n}$ is the unit vector along the direction of wave propagation, the symbol $x$ represents either the pump laser (when $x$ is substituted by $p$ ) or the probe laser (when $x$ is substituted by $t$ ), and $\omega_{x}$ and $A_{x}$ are the frequency and amplitude of the corresponding laser, respectively. Accordingly, Hamiltonian that describes the interaction of the electron and the electromagnetic field accompanying the laser can be written as [50]

$$
\widehat{H}_{\mathrm{int}}=-\frac{e}{m_{0}} \frac{A_{x} \cdot e^{-i \omega_{x} t}}{i \omega_{x}} \vec{n} \widehat{\vec{p}}
$$

where $m_{0}$, e, and $\vec{p}$ represent the bare mass, charge, and momentum of electron, respectively.

When the strong resonant pump laser is switched on, the wave functions of the electron are renormalized. Now, the electrons stay in a superposition state represented by the wave function

$$
\begin{aligned}
\Pi_{\text {mix }}^{e}(\vec{r}, t)= & \frac{1}{2 \Omega_{R}}\left(\alpha_{1} e^{-(i / \hbar) E_{1}^{-} t}+\alpha_{2} e^{-(i / \hbar) E_{1}^{+} t}\right) \Pi_{1}(\vec{r}) \\
& -\frac{V_{21}}{2 \hbar \Omega_{R}}\left(e^{-(i / \hbar) E_{2}^{-} t}-e^{-(i / \hbar) E_{2}^{+} t}\right) \Pi_{2}(\vec{r}),
\end{aligned}
$$

where $\Pi_{1}(\vec{r})$ and $\Pi_{2}(\vec{r})$ are the initial electron wave functions in the absence of the pump laser as given in Equation (7). When the pump laser operates, the energy spectrum of the electron are expanded to four levels in which two new levels $E_{1}^{-}$and $E_{1}^{+}$come from the splitting of the initial level
$E_{1}$, and two sublevels $E_{2}^{-}$and $E_{2}^{+}$are detached from the initial level $E_{2}$ (as plotted in Figure 1(b)). These splitting levels are

$$
\begin{gathered}
\left\{\begin{array}{l}
E_{1}^{-}=E_{1}-\hbar \alpha_{2}, \\
E_{1}^{+}=E_{1}+\hbar \alpha_{1},
\end{array}\right. \\
\left\{\begin{array}{l}
E_{2}^{-}=E_{2}-\hbar \alpha_{1}, \\
E_{2}^{+}=E_{2}+\hbar \alpha_{2} .
\end{array}\right.
\end{gathered}
$$

In Equation (10), we set

$$
\left\{\begin{array}{l}
\alpha_{1}=\Omega_{R}-\frac{\Delta \omega}{2}, \\
\alpha_{2}=\Omega_{R}+\frac{\Delta \omega}{2}, \\
\Omega_{R}=\sqrt{\frac{\Delta \omega^{2}}{4}+\frac{\left|V_{21}\right|^{2}}{\hbar^{2}}},
\end{array}\right.
$$

with $\hbar \Delta \omega$ being the pump laser detuning, which is the energy difference between the photon energy of the pump laser and the energy distance between two original electron levels $E_{1}$ and $E_{2}, \hbar \Delta \omega=\hbar \omega_{p}-\left(E_{2}-E_{1}\right)$; please look at the left side of Figure 1(b) for a schematic view of the pump laser detuning. In addition, $V_{21}$ is the intraband transition matrix element between those levels:

$$
V_{21}=\frac{e}{m_{0}} \frac{A_{p}}{i \omega_{p}} \frac{m_{e}}{i \hbar} \frac{\left(E_{2}-E_{1}\right) R}{J_{1}\left(\chi_{01}\right) J_{2}\left(\chi_{11}\right)} \int_{0}^{1} J_{0}\left(\chi_{01} r\right) J_{1}\left(\chi_{11} r\right) r^{2} d r
$$

where $m_{e}$ is the effective mass of electron. We see that the matrix element depends not only on the parameters of the quantum wire but also on those of the pump laser.

2.3. The Absorption Spectrum of Excitons in the Absence of the Pump Laser. We will analyze the exciton absorption spectrum in quantum wires to study the excitonic optical Stark effect. In order to determine the exciton absorption spectrum, we have to calculate the transition rate through the interband transition matrix element from the level of the hole to levels of electrons. First, we will investigate the case in which the system is under no pump laser. In this case, due to the selection rules for the quantum wire structure, there is only a possible occurrence of the interband transition between the hole level $E_{0}$ and the first electron level $E_{1}$ under the excitation of a suitable probe laser, as sketched by the thin arrow in Figure 1(a). The matrix element of the interband transition between these levels is determined as follows:

$$
T_{10}=\left\langle\Pi_{1}(\vec{r}, t)\left|\widehat{H}_{\mathrm{int}}\right| \Pi_{0}(\vec{r}, t)\right\rangle
$$


By substituting Equations (6) and (9) into Equation (15), we have

$$
T_{10}=-\frac{e A_{t}}{m_{0} i \omega_{t}} e^{(i / \hbar)\left(E_{1}-E_{0}-\hbar \omega_{t}\right) t}\left\langle\Pi_{1}(\vec{r})|\vec{n} \widehat{\vec{p}}| \Pi_{0}(\vec{r})\right\rangle .
$$

By using Equation (7), we can rewrite Equation (16) as follows:

$$
\begin{aligned}
T_{10}= & -\frac{e A_{t}}{m_{0} i \omega_{t}} e^{(i / \hbar)\left(E_{1}-E_{0}-\hbar \omega_{t}\right) t}\left\langle u_{c}(\vec{r})|\vec{n} \widehat{\vec{p}}| u_{v}(\vec{r})\right\rangle \\
& \cdot\left\langle\Psi_{01}^{e}(\vec{r}) \mid \Psi_{01}^{h}(\vec{r})\right\rangle,
\end{aligned}
$$

or

$$
T_{10}=-\frac{e A_{t} p_{\mathrm{cv}}}{m_{0} i \omega_{t}} e^{(i / \hbar)\left(E_{1}-E_{0}-\hbar \omega_{t}\right) t},
$$

where $\omega_{t}$ and $A_{t}$ are the frequency and amplitude of the probe laser, respectively, and $p_{\mathrm{cv}}$ is the polarization matrix element between Bloch functions of the conduction and the valence band

$$
p_{c v}=\left\langle u_{c}(\vec{r})|\vec{n} \widehat{\vec{p}}| u_{v}(\vec{r})\right\rangle .
$$

From Equation (18), in the absence of the pump laser, we find the transition rate as follows [51]:

$$
W_{0}=\frac{2 \pi}{\hbar}\left(\frac{e A_{t} p_{c v}}{m_{0} \omega_{t}}\right)^{2} \delta\left(E_{1}-E_{0}-\hbar \omega_{t}\right)
$$

The transition rate can be rewritten in Lorentz's form as follows [52]:

$$
W_{0}=\frac{2}{\hbar}\left(\frac{e A_{t} p_{c v}}{m_{0} \omega_{t}}\right)^{2} \frac{\Gamma}{\left(E_{1}-E_{0}-\hbar \omega_{t}\right)^{2}+\Gamma^{2}},
$$

where $\Gamma$ is the phenomenological linewidth of the absorption peak.

2.4. The Exciton Absorption Spectrum in the Presence of the Pump Laser. Now, we will calculate the interband transition rate when the pump laser operates. As mentioned above, under the effect of a strong resonant pump laser, the electrons are now in the superposition state specified by the renormalized wave function given in Equation (10). Then, the matrix element for the interband transition between the hole state and the electron superposition state is determined as follows:

$$
T_{\text {mix }, 0}=\left\langle\Pi_{\text {mix }}^{e}(\vec{r}, t)\left|\widehat{H}_{\text {int }}\right| \Pi_{0}(\vec{r}, t)\right\rangle .
$$

Replacing Equations (6), (9), and (10) into Equation (22), we have

$$
\begin{aligned}
T_{\mathrm{mix}, 0}= & -\frac{e A_{t} e^{-i \omega_{t} t}}{m_{0} i \omega_{t}}\left[\frac{1}{2 \Omega_{R}}\left(\alpha_{1} e^{-(i / \hbar) E_{1}^{-} t}+\alpha_{2} e^{-(i / \hbar) E_{1}^{+} t}\right)^{*} e^{-(i / \hbar) E_{0} t}\right. \\
& \cdot\left\langle\Pi_{1}(\vec{r})|\vec{n} \widehat{\vec{p}}| \Pi_{0}(\vec{r})\right\rangle-\frac{V_{21}}{2 \hbar \Omega_{R}} \\
& \cdot\left(e^{-(i / \hbar) E_{2}^{-} t}-e^{-(i / \hbar) E_{2}^{+} t}\right)^{*} e^{-(i / \hbar) E_{0} t} \\
& \left.\cdot\left\langle\Pi_{2}(\vec{r})|\vec{n} \widehat{\vec{p}}| \Pi_{0}(\vec{r})\right\rangle\right] .
\end{aligned}
$$

Notably, when the probe laser irradiates on the system, due to the selection rules for the quantum wire structure, the interband transitions can only occur among the hole level $E_{0}$ and the electron splitting levels $E_{1}^{-}$and $E_{1}^{+}$(thin arrows in Figure 1(b)). Therefore, in Equation (23), we can omit the second term and rewrite it as follows:

$$
\begin{aligned}
T_{\text {mix }, 0}= & -\frac{e A_{t} e^{-i \omega_{t} t}}{m_{0} i \omega_{t}}\left[\frac{1}{2 \Omega_{R}}\left(\alpha_{1} e^{-(i / \hbar) E_{1}^{-} t}+\alpha_{2} e^{-(i / \hbar) E_{1}^{+} t}\right)^{*} e^{-(i / \hbar) E_{0} t}\right. \\
& \left.\cdot\left\langle\Pi_{1}(\vec{r})|\vec{n} \overrightarrow{\vec{p}}| \Pi_{0}(\vec{r})\right\rangle\right] .
\end{aligned}
$$

Substituting Equation (7) into Equation (24), we obtain

$$
\begin{aligned}
T_{\text {mix }, 0}= & -\frac{e A_{t} p_{\mathrm{cv}}}{m_{0} i \omega_{t}}\left[\frac{1}{2 \Omega_{R}}\left(\alpha_{1} e^{(i / \hbar)\left(E_{1}^{-}-E_{0}-\hbar \omega_{t}\right) t}+\alpha_{2} e^{(i / \hbar)\left(E_{1}^{+}-E_{0}-\hbar \omega_{t}\right) t}\right)\right. \\
& \left.\cdot\left\langle\Psi_{01}^{e}(\vec{r}) \mid \Psi_{01}^{h}(\vec{r})\right\rangle\right],
\end{aligned}
$$

or

$$
T_{\text {mix }, 0}=-\frac{e A_{t} p_{\mathrm{cv}}}{m_{0} i \omega_{t}}\left[\frac{\alpha_{1}}{2 \Omega_{R}} e^{(i / \hbar)\left(E_{1}^{-}-E_{0}-\hbar \omega_{t}\right) t}+\frac{\alpha_{2}}{2 \Omega_{R}} e^{(i / \hbar)\left(E_{1}^{+}-E_{0}-\hbar \omega_{t}\right) t}\right] .
$$

Then, we can determine the interband transition rate under the effect of a resonant pump laser as [51]

$$
\begin{aligned}
W= & \frac{2 \pi}{\hbar}\left(\frac{e A_{t} p_{\mathrm{cv}}}{m_{0} \omega_{t}}\right)^{2}\left[\left(\frac{\alpha_{1}}{2 \Omega_{R}}\right)^{2} \delta\left(E_{1}^{-}-E_{0}-\hbar \omega_{t}\right)\right. \\
& \left.+\left(\frac{\alpha_{2}}{2 \Omega_{R}}\right)^{2} \delta\left(E_{1}^{+}-E_{0}-\hbar \omega_{t}\right)\right] .
\end{aligned}
$$

We omit the crossing terms in the above formula due to the negligible overlap of the delta functions. Then, by 
applying a similar transformation as in the previous section, we get a clearer form of the transition rate as

$$
\begin{aligned}
W=\frac{2}{\hbar}\left(\frac{e A_{t} p_{\mathrm{cv}}}{m_{0} \omega_{t}}\right)^{2}\left[\left(\frac{\alpha_{1}}{2 \Omega_{R}}\right)^{2} \frac{\Gamma}{\left(E_{1}^{-}-E_{0}-\hbar \omega_{t}\right)^{2}+\Gamma^{2}}\right. \\
\left.+\left(\frac{\alpha_{2}}{2 \Omega_{R}}\right)^{2} \frac{\Gamma}{\left(E_{1}^{+}-E_{0}-\hbar \omega_{t}\right)^{2}+\Gamma^{2}}\right] .
\end{aligned}
$$

where $\Gamma$ is the linewidth of the absorption peak described in the previous section. As calculated from Fermi's golden rule, the transition rate $W$ is the probability of transition per unit time from the initial state to the final state, so its unit is the number of transitions per unit time. In this study, however, we will consider $W$ in the arbitrary unit for the sake of convenience.

\section{Results and Discussion}

In this section, we study the exciton absorption spectrum in $\mathrm{GaAs} / \mathrm{Al}_{0.7} \mathrm{Ga}_{0.3} \mathrm{As}$ cylindrical quantum wires in the absence and in the presence of the pump laser to reveal the excitonic optical Stark effect. Of course, the band offset between GaAs and $\mathrm{Al}_{0.7} \mathrm{Ga}_{0.3} \mathrm{As}$ is finite, but here, in our very first work related to quantum wires, we assume quantum wires being surrounded by an infinite potential for the sake of simplicity. Therefore, we do not mention the effect of the barrier layer in the later discussion but leave it for future work. The parameters are used as follows. The pump laser is assigned an amplitude of about $A_{p}=8 \times 10^{6} \mathrm{~V} / \mathrm{m}$ and has a very small detuning, $\hbar \Delta \omega \ll \hbar \omega_{p}$. The linewidth of the absorption peak is assumed to be $\Gamma=0.1 \mathrm{meV}$; here, we use a monochrome probe laser. The effective masses of the electron and hole of the wire material, GaAs, are $m_{e}=0.067 m_{0}$ and $m_{h}=0.51 m_{0}$, respectively [53]; the energy gap of GaAs is $E_{g}=1424 \mathrm{meV}$ [54].

First, we plot the absorption spectra of excitons in the quantum wire of the radius $R=50 \AA$ in the absence and in the presence of a strong pump laser that resonates accurately with two levels of the electron $(\hbar \Delta \omega=0 \mathrm{meV})$, Figure 2 . The results show that, before the pump laser operates, the absorption spectra of excitons include only one absorption peak (dashed line). By contrast, after turning on the pump laser, two distinct peaks (solid lines) arise in the exciton absorption spectra as convincing evidence of the existence of the excitonic optical Stark effect in the system. These results can be explained as follows.

Before the pump laser is switched on, according to the selection rules for the quantum wire structures, there is only one transition between the hole level and the first electron level in the absorption spectrum of excitons (the thin arrow in Figure 1(a)). Therefore, we see only one peak in the absorption spectrum. On the contrary, if there exists a resonant pump laser that can excite the intraband transition between two electron levels (as illustrated by the thick arrow in Figure 1(b)), and if the intensity of the pump laser is very strong, then it can merge those two electron levels into a single level. According to the Pauli exclusion principle, this sin-

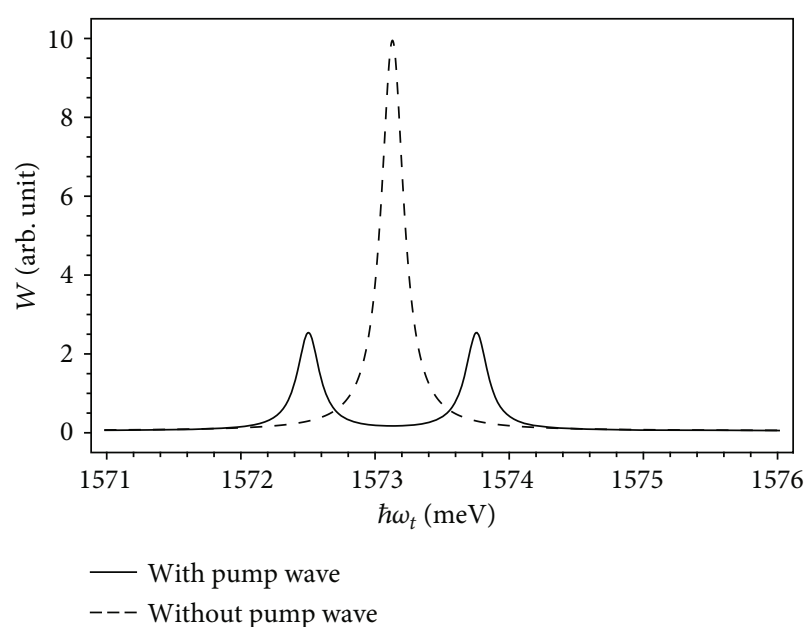

FIgURE 2: The exciton absorption spectra in the cylindrical quantum wire with radius $R=50 \AA$ in the absence (dashed line) and in the presence (solid line) of the pump laser with the detuning $\hbar \Delta \omega=0 \mathrm{meV}$.

gle level allows a maximum of two states of electron, while two initial electron levels allow up to four states. In order to ensure that the system still includes the four permitted states and still satisfies the Pauli exclusion principle, each initial electron level needs to be split into two sublevels. Fortunately, under the strong electric field of the pump laser, the symmetry of the state of this single level is broken as seen in the Stark effect. Therefore, both initial electron levels are split. Consequently, when we irradiate a proper probe laser into the system, we can find two interband transitions from the hole level to two sublevels of electron (as indicated by the two thin arrows in Figure 1(b)), and thus, we can see two distinct peaks in the exciton absorption spectrum. Obviously, due to the selection rules, it is impossible that the interband transitions from the hole level to the electron sublevels split from the second quantized level of the electron will occur. Moreover, due to the symmetry between the sublevels with reference to the position of the initial electron level, the two obtained peaks have the same height (or the same transition rate) and stay symmetrical on both sides of the initial peak, which satisfies the energy conservation law. We also found that, in Figure 2, the intensity of the exciton absorption spectra in the case of without pump wave (dashed line) appears much higher than that in the presence of the pump wave (solid line). This reduction is due to the act of the conservation of the transition rate [55]. We realize that, by turning the pump laser on or off, this effect can control the system to work as an optical switching by letting the probe laser beam of certain frequency propagate through it or not.

Now, we will thoroughly study the excitonic optical Stark effect in the quantum wires by examining the dependence of the exciton absorption spectrum on the pump laser detuning and the wire radius. First, we plot the exciton absorption spectra in the quantum wire with radius $R=50 \AA$ with different detuning in both cases with and without the pump laser (Figure 3). We observe that in the presence of the pump laser, all excitonic absorption spectra contain two distinct spectral 


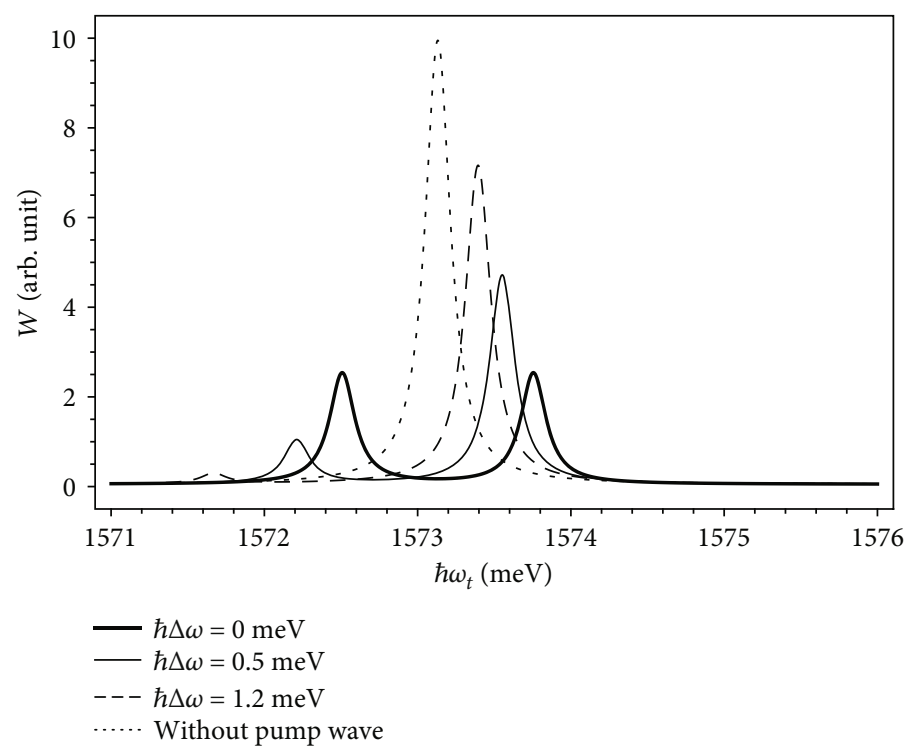

FIgURE 3: The exciton absorption spectra in cylindrical quantum wire of radius $R=50 \AA$ in the absence (dotted line) and in the presence of the resonant pump laser with specific detuning $\hbar \Delta \omega=0 \mathrm{meV}$ (thick solid line); $\hbar \Delta \omega=0.5 \mathrm{meV}$ (thin solid line); $\hbar \Delta \omega=1.2 \mathrm{meV}$ (dashed line).

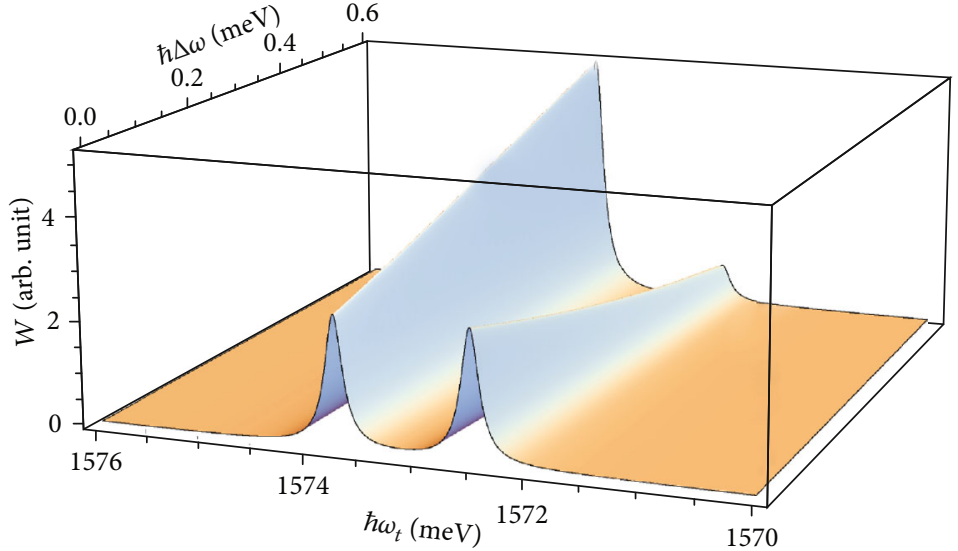

FIgURE 4: The exciton absorption spectra in the cylindrical quantum wire of radius $R=50 \AA$ as a function of the photon energy of the probe laser $\hbar \omega_{t}$ and the detuning $\hbar \Delta \omega$ of the pump laser.

peaks, which implies that the optical Stark effect occurs. Moreover, as we increase the detuning, the height of one peak increases and that peak moves closer to the position of the initial peak, while the other peak gets farther from the position of the initial peak and lowered, and it almost disappears when the detuning is too large (as in the case $\hbar \Delta \omega=1.2 \mathrm{meV}$, dashed line). The higher the difference in height of the two spectral peaks is, the greater the difference in the transition rate. Despite this, in all the cases under investigation, we can see that the total transition rate is conserved.

Figure 4 shows more clearly the change in height and position of the absorption peaks when the pump laser detuning is increased from $0 \mathrm{meV}$ to $0.6 \mathrm{meV}$. It is obvious that when the detuning increases, then one peak raises its height while the other lowers to the zero value.

Next, we investigate the influence of the quantum wire radius on the excitonic optical Stark effect. Figure 5 shows the exciton absorption spectra in the quantum wires of radii
$R=45 \AA$ (solid line), $R=50 \AA$ (dashed line), and $R=55 \AA$ (dotted line) in the presence of the pump laser with the detuning $\hbar \Delta \omega=0.5 \mathrm{meV}$. In all three cases, we observe the appearance of two exciton absorption peaks, which again confirmed the existence of the optical Stark effect. We can see that as the wire radius increases, the absorption spectra of excitons shift very fast to the lower energy region. This can be explained as follows. According to Equation (4), as the quantum wire radius increases, the quantized levels of the electron and hole have smaller values and they stay closer to each other. Therefore, a smaller photon energy is needed to excite the transition from the hole level to the splitting electron levels. As a result, we see that the absorption spectra of the excitons shift to a lower energy region as the wire radius becomes larger. It is worth mentioning that we have applied our theory for the other low-dimensional structures such as quantum dots and quantum wells and we have obtained the similar results $[55,56]$. 


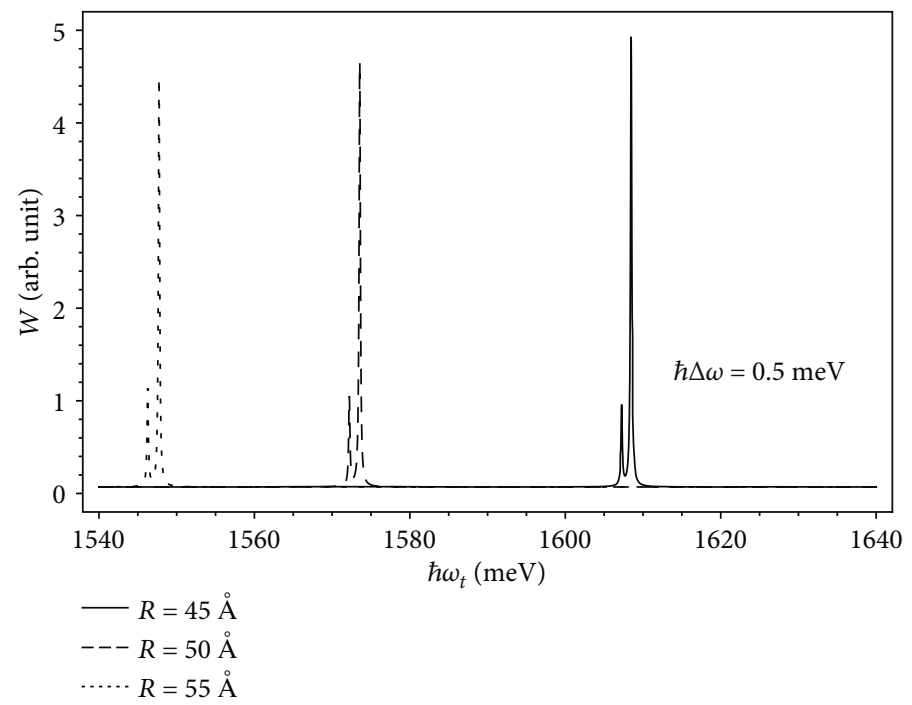

FIgURE 5: The absorption spectra of excitons in the cylindrical quantum wires with various radii in the presence of pump laser with the detuning $\hbar \Delta \omega=0.5 \mathrm{meV}$.

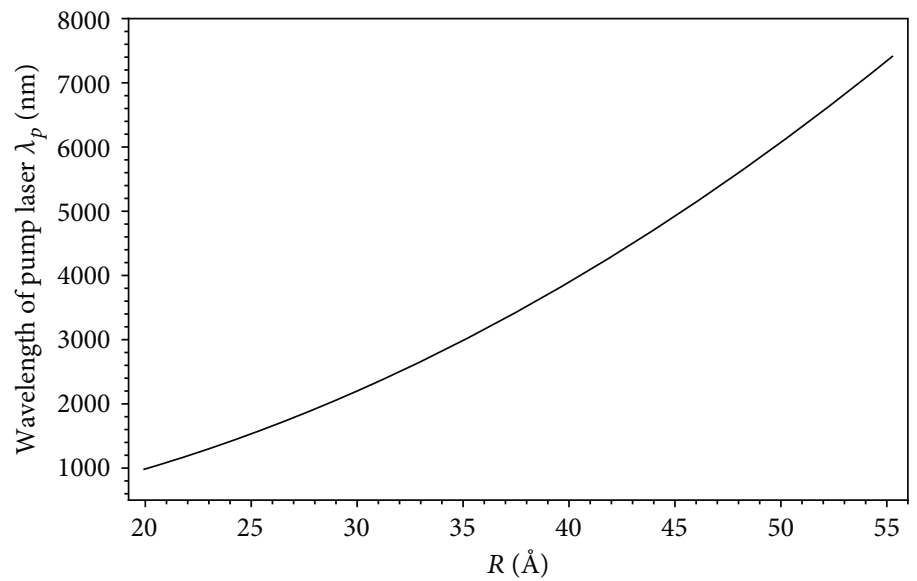

FIGURE 6: The wavelength of pump laser of zero detuning as a function of wire radius.

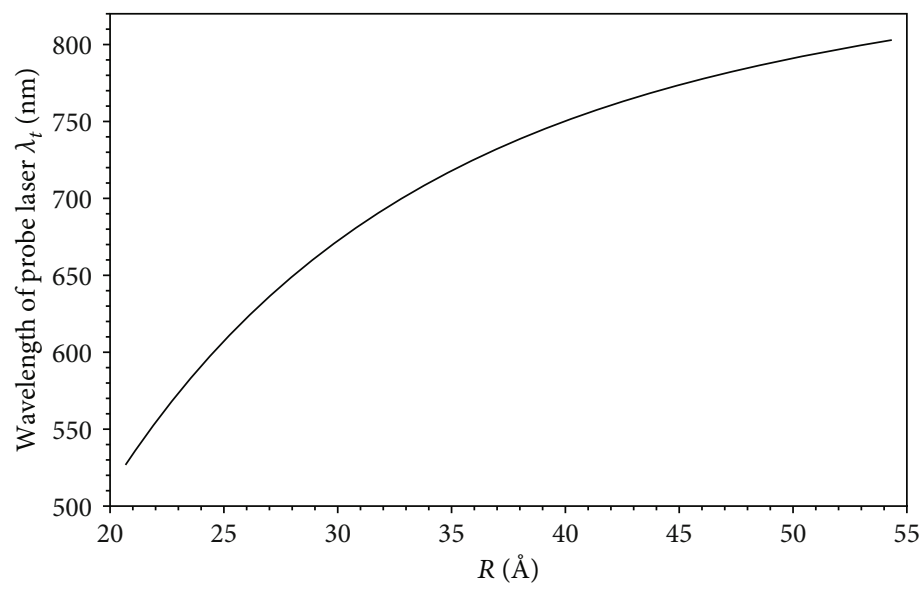

FIGURE 7: The wavelength of probe laser $\lambda_{t}$ as a function of wire radius.

As an effort to provide a brief guidance for experimental verification of our results, in Figures 6 and 7, the dependence of the wavelength of pump laser $\lambda_{p}$ and that of probe laser $\lambda_{t}$ on the wire radius are plotted. We see that both the wavelengths increase with the wire radius as a result of the quantum size effect. However, while one curve describes a 
parabolic function (Figure 6), the other shows a square root one (Figure 7).

After fabricating quantum wires, by looking at these figures, experimentalists can find the proper wavelengths of lasers and set up verification experiments. By controlling the laser's powers to fit values, the excitonic optical Stark effect is expected to occur. Also, by adjusting the other parameters, we can find the properties of the excitonic optical Stark effect.

\section{Conclusions}

In this work, we have studied the three-level excitonic optical Stark effect in GaAs circular cylindrical quantum wires by means of the renormalized wave function theory. The results show that, while the pump laser operates, there appear two separated spectral peaks in the excitonic absorption spectra as the indication of the excitonic optical Stark effect. Moreover, the absorption spectra of excitons shift sharply with the wire radius. Also, the heights of the two absorption peaks are very sensitive to the detuning of the pump laser.

Additionally, we have proposed an explanation for the splitting of the energy levels of the electron under the effect of the strong resonant pump laser, which leads to the formation of two new absorption peaks of the exciton. We have also tried to explain the shift of the excitonic absorption spectra as the wire radius varies. Finally, we have tried to provide a brief guidance for experimental verification of our results. We expect that these results could be confirmed by proper experiments and could have some contribution to the related fields such as making ultrafast switching devices for quantum computers.

For the sake of simplicity, here, we consider circular cylindrical quantum wires surrounded by an infinite potential. However, we believe that our model can be expanded to other quantum wires of different shapes under various potentials as long as we can determine the wave functions and energy levels of particles.

\section{Data Availability}

The material structure parameters used to support the findings of this study are included within the article.

\section{Conflicts of Interest}

The authors declare that they have no conflicts of interest.

\section{Acknowledgments}

This research is funded by the Vietnam National Foundation for Science and Technology Development (NAFOSTED) under grant number 103.01-2017.321.

\section{References}

[1] H. Cansizoglu, E. Ponizovskaya Devine, Y. Gao et al., "A new paradigm in high-speed and high-efficiency silicon photodiodes for communication-part I: enhancing photon-material interactions via low-dimensional structures," IEEE Transactions on Electron Devices, vol. 65, no. 2, pp. 372-381, 2018.

[2] B. Kelleher, C. Bonatto, G. Huyet, and S. P. Hegarty, "Excitability in optically injected semiconductor lasers: contrasting quantum-well- and quantum-dot-based devices," Physical Review E, vol. 83, no. 2, article 026207, 2011.

[3] R. D. Dupuis, "III-V semiconductor quantum-well devices grown by metalorganic chemical vapor deposition," Proceedings of the IEEE, vol. 101, no. 10, pp. 2188-2199, 2013.

[4] J. Y. Huang, C. Y. Lin, W.-S. Liu, and J.-I. Chyi, "Quantum control study of ultrafast optical responses in semiconductor quantum dot devices," Optics Express, vol. 22, no. 25, pp. 30815-30825, 2014.

[5] M. K. Yakes, N. Mahadik, S. B. Qadri et al., "Scanning probe microscopy based characterizations of III-V semiconductor quantum well devices," Microscopy and Microanalysis, vol. 18, no. S2, pp. 1810-1811, 2012.

[6] S. Iizuka, H. Asai, J. Hattori, K. Fukuda, and T. Mori, "Implementation of Coulomb blockade transport on a semiconductor device simulator and its application to tunnel-FET-based quantum dot devices," Japanese Journal of Applied Physics, vol. 59, no. SI, article SIIE02, 2020.

[7] M. G. Barseghyan, C. A. Duque, E. C. Niculescu, and A. Radu, "Intense laser field effects on the linear and nonlinear optical properties in a semiconductor quantum wire with triangle cross section," Superlattices and Microstructures, vol. 66, pp. 10-22, 2014.

[8] S. Saravanan, A. J. Peter, and C. W. Lee, "Laser field induced optical gain in a group III-V quantum wire," The European Physical Journal D, vol. 70, no. 8, p. 165, 2016.

[9] Y. K. Ezhovskii, "Chemical nanotechnology of oxide and nitride low-dimensional structures on a semiconductor matrix," Russian MicroElectronics, vol. 39, no. 3, pp. 182189,2010

[10] H. Hardtdegen, M. Mikulics, S. Rieß et al., "Modern chemical synthesis methods towards low-dimensional phase change structures in the Ge-Sb-Te material system," Progress in Crystal Growth and Characterization of Materials, vol. 61, no. 2-4, pp. 27-45, 2015.

[11] F. J. Owens, The Physics of Low Dimensional Materials, World Scientific Publishing Company, 2017.

[12] Y. Sun, K. Liu, and Y. Zhu, "Recent progress in synthesis and application of low-dimensional silicon based anode material for lithium ion battery," Journal of Nanomaterials, vol. 2017, Article ID 4780905, 15 pages, 2017.

[13] K. Boeneman, D. E. Prasuhn, J. B. Blanco-Canosa et al., "Quantum dots as a FRET donor and nanoscaffold for multivalent DNA photonic wires," in Proceedings of SPIE, vol. 7909 of Colloidal Quantum Dots/Nanocrystals for Biomedical Applications VI, p. 79090R, San Francisco, CA, USA, 2011.

[14] P. Bhattacharya, A. D. Stiff-Roberts, S. Krishna, and S. Kennerly, "Quantum dot infrared detectors and sources," International Journal of High Speed Electronics and Systems, vol. 12, pp. 969-994, 2011.

[15] A. P. Litvin, I. V. Martynenko, F. Purcell-Milton, A. V. Baranov, A. V. Fedorov, and Y. K. Gun'ko, "Colloidal quantum dots for optoelectronics," Journal of Materials Chemistry A, vol. 5, no. 26, pp. 13252-13275, 2017.

[16] A. Boutramine, A. Nafidi, D. Barkissy, A. Hannour, A. Elanique, and T. E. Gouti, "Application of the transition semiconductor to semimetal in type II nanostructure 
superlattice for mid-infrared optoelectronic devices," Applied Physics A, vol. 122, no. 4, 2016.

[17] S. G. Schirmer and P. J. Pemberton-Ross, "Fast high-fidelity information transmission through spin-chain quantum wires," Physical Review A, vol. 80, no. 3, article 030301, 2009.

[18] R. Castellanos, R. Franco, J. Silva-Valencia, and M. S. Figueira, "Thermoelectric transport properties of a quantum wire, coupled to a quantum dot: finite band effects," Journal of Superconductivity and Novel Magnetism, vol. 23, no. 1, pp. 153-155, 2010.

[19] R. Khordad, "Quantum wire with parallelogram cross section: optical properties," Journal of Theoretical and Applied Physics, vol. 6, no. 1, p. 19, 2012.

[20] H. Bahramiyan and R. Khordad, "The effect of electronphonon interaction on optical properties of a triangular quantum wire," Superlattices and Microstructures, vol. 63, pp. 267276, 2013.

[21] R. Khordad and H. Bahramiyan, "Effect of size distribution on the optical properties of quantum wire systems," International Journal of Modern Physics, vol. 28, no. 18, article 1450119, 2014.

[22] R. Khordad and H. R. Rastegar Sedehi, "Low temperature behavior of thermodynamic properties of 1D quantum wire under the Rashba spin-orbit interaction and magnetic field," Solid State Communications, vol. 269, pp. 118-124, 2018.

[23] D. Piester, A. A. Ivanov, A. S. Bakin, H.-H. Wehmann, and A. Schlachetzki, "Semiconductor nanostructures for quantum wire lasers," Proceedings of SPIE, vol. 4748, pp. 476-485, 2001.

[24] S. Arai and T. Maruyama, "GaInAsP/InP quantum wire lasers," IEEE Journal of Selected Topics in Quantum Electronics, vol. 15, no. 3, pp. 731-742, 2009.

[25] M. A. Grado-Caffaro and M. Grado-Caffaro, "Electron wavelength and drift mobility operators for treating quantum electron waveguides," Optik, vol. 125, no. 21, pp. 6543-6544, 2014.

[26] J. B. Khurgin and D. Yang, "Resonant tunneling field-effect transistor based on wave function shape modulation in quantum wires," Journal of Applied Physics, vol. 85, no. 6, pp. 32183221, 1999.

[27] D. Yang and J. B. Khurgin, "Analysis of the performance of the quantum wire resonant tunneling field-effect transistor," Superlattices and Microstructures, vol. 27, no. 4, pp. 245-254, 2000.

[28] S. Saravanan and A. John Peter, "Binding energy of a magnetoexciton in an InAsP quantum well wire for the potential application of telecommunication networks," Materials Today: Proceedings, vol. 2, pp. 4373-4377, 2015.

[29] L. Donaldson, "Quantum effects in 1D wires," Materials Today, vol. 19, no. 10, pp. 550-551, 2016.

[30] K. Tai, J. Hegarty, and W. T. Tsang, "Observation of optical Stark effect in InGaAs/InP multiple quantum wells," Applied Physics Letters, vol. 51, no. 3, pp. 152-154, 1987.

[31] T. Unold, K. Mueller, C. Lienau, T. Elsaesser, and A. D. Wieck, "Optical Stark effect in a quantum dot: ultrafast control of single exciton polarizations," Physical Review Letters, vol. 92, no. 15, 2004.

[32] M. Combescot and R. Combescot, "Optical Stark effect of the exciton: biexcitonic origin of the shift," Physical Review B, vol. 40, no. 6, pp. 3788-3801, 1989.

[33] I. M. Gadzhiyev, M. S. Buyalo, A. E. Gubenko et al., "Switching between the mode-locking and Q-switching modes in twosection QW lasers upon a change in the absorber properties due to the Stark effect," Semiconductors, vol. 50, no. 6, pp. 828-831, 2016.

[34] D. Ahn, "Enhancement of the Stark effect in coupled quantum wells for optical switching devices," IEEE Journal of Quantum Electronics, vol. 25, no. 11, pp. 2260-2265, 1989.

[35] Y. Rong, Y. Huo, E. T. Fei et al., "High speed optical modulation in Ge quantum wells using quantum confined Stark effect," Frontiers of Optoelectronics, vol. 5, no. 1, pp. 82-89, 2012.

[36] M. Gogoi, P. Deb, D. Sen, S. Mazumder, and A. Kostka, "Enhanced quantum confined Stark effect in a mesoporous hybrid multifunctional system," Solid State Communications, vol. 187, pp. 48-52, 2014.

[37] J. T. Liu, F. H. Su, H. Wang, and X. H. Deng, "The influence of the optical Stark effect on chiral tunneling in graphene," EPL (Europhysics Letters), vol. 95, no. 2, article 24003, 2011.

[38] E. I. Garmire, A. I. A. Maradudin, and K. K. Rebane, Laser Optics of Condensed Matter (Volume 2 the Physics of Optical Phenomena and Their Use as Probes of Matter), Springer Science+Business Media, LCC, 1991.

[39] D. Fröhlich, C. Neumann, B. Uebbing, and R. Wille, "Experimental investigation of three-level optical Stark effect in semiconductors," Physica Status Solidi (B), vol. 159, no. 1, pp. 297 307, 1990.

[40] D. Song, F. Wang, G. Dukovic et al., "Measurement of the optical Stark effect in semiconducting carbon nanotubes," Applied Physics A: Materials Science \& Processing, vol. 96, no. 2, pp. 283-287, 2009.

[41] S. Efumi, Y. Uchibori, J. Ishihara, and K. Miyajima, "Observation of optical Stark effect between $1 s$ - 2pexciton levels in $\mathrm{CuCl}$ single crystal," Journal of Physics: Conference Series, vol. 1220, p. $012022,2019$.

[42] N. H. Quang, "The optical Stark effect of the exciton due to dynamical coupling between quantized states of the electron and hole in quantum wells," International Journal of Modern Physics B, vol. 7, pp. 3405-3413, 1993.

[43] S. Zielińska-Raczyńska, G. Czajkowski, and D. Ziemkiewicz, "Quantum confined Stark effect in wide parabolic quantum wells: real density matrix approach," The European Physical Journal B, vol. 88, no. 12, article 338, 2015.

[44] T. Altevogt and R. Zimmermann, "Nonclassical gain in the optical Stark effect: density matrix approach," Journal of Physics B: Atomic, Molecular and Optical Physics, vol. 32, no. 19, pp. 4719-4728, 1999.

[45] S. Wang, Y. Kang, and C. Han, "Transverse Stark effect in the optical absorption in a square semiconducting quantum wire," Journal of Semiconductors, vol. 34, no. 10, article 102001, 2013.

[46] A. Rustagi and A. F. Kemper, "Coherent excitonic quantum beats in time-resolved photoemission measurements," Physical Review B, vol. 99, no. 12, article 125303, 2019.

[47] D. Sangalli, E. Perfetto, G. Stefanucci, and A. Marini, "An abinitio approach to describe coherent and non-coherent exciton dynamics," The European Physical Journal B, vol. 91, no. 8, article 171, 2018.

[48] D. D. Phuoc, L. T. N. Bao, L. T. D. Hien, H. K. Hieu, and D. N. Thao, "A study on quantum beats of excitons in GaAs/AlGaAs circular cylindrical quantum wires," Japanese Journal of Applied Physics, vol. 59, no. 12, article 125003, 2020.

[49] L. Wendler and V. G. Grigoryan, "Effect of the image potential on plasmons in cylindrical quantum-well wires," Physica Status Solidi (B), vol. 181, no. 1, pp. 133-159, 1994. 
[50] E. Rosencher, B. Vinter, and P. G. Piva, Optoelectronics, Cambridge University Press, 2002.

[51] N. Zettili, Quantum Mechanics: Concepts and Applications, Wiley, 2nd ed edition, 2009.

[52] V. Balakrishnan, "All about the Dirac delta function (?)," Resonance, vol. 8, no. 8, pp. 48-58, 2003.

[53] W. Nakwaski, "Effective masses of electrons and heavy holes in GaAs, InAs, A1As and their ternary compounds," Physica B, vol. 210, no. 1, pp. 1-25, 1995.

[54] J. S. Blakemore, "Semiconducting and other major properties of gallium arsenide," Journal of Applied Physics, vol. 53, no. 10, pp. R123-R181, 1982.

[55] D. N. Thao, L. T. N. Bao, D. D. Phuoc, and N. H. Quang, "A theoretical study of the optical Stark effect in InGaAs/InAlAs quantum dots," Semiconductor Science and Technology, vol. 32, no. 2, article 025014, 2017.

[56] L. T. N. Bao and D. N. Thao, "Theoretical investigation of quantum beat of excitons in GaAs/AlGaAs quantum wells," in 42nd Vietnam National Conference on Theoretical Physics (NCTP-42), Cantho, Vietnam, 2017. 